\title{
4
}

\section{Powerline Communication in Home-Building Automation Systems}

\author{
Elena Mainardi and Marcello Bonfè \\ Engineering Department, University of Ferrara \\ Italy
}

\section{Introduction}

Domotics, Smart Home Systems, Ambient Intelligence are all terms that describe the intelligent cooperation of several different equipments to manage the home environment in an intelligent, safe and comfortable way. The same idea is also applicable to bigger constructions, and in that case it takes the name of Building Automation.

Whatever term one wants to use, it refers to a multidisciplinary field that includes informatics, electronics, automation and telecommunication, and also touches fields like building constructions and architecture. In fact, during the process of designing a building, people have to consider appropriate spaces for the electric plant, and if the presence of a domotic system is planned, it is better to take it into account during the design phase, just to optimize spaces, the amount of used wires, the position of the modules and so on.

There are really many home system producers in the world (Smart Home Systems, EIBKonnex, Lonworks, Bticino, Vimar, Duemmegi, EasyDom Corporation, Futurware, Digital Cybermasters, Hills Home Systems, Intellihome etc, just to mention a few), and their products differ from each others in many characteristics, such as functionality, dimension, weight, typology of installation, materials, net topology, power consumes, aesthetic appearance, communication protocol and communication mean.

Regarding this last point, the majority of the domotic systems, especially in Europe, tend to use a dedicated bus cable to exchange data among modules, to make the communication link more robust and reliable. Lately, using radio communication is in fashion, but radio modules, respect to their equivalent standard ones, are more expensive, and in the bargain many people don't want to use them due to the fear of radio signals (even if it were proved that they are completely harmless).

Another communication mean, that is often not taken into account, is the powerline.

In point of fact, using the installed poweline wires to send information is a very smart idea: there is no additional cost to install other dozens of meters of wires, there is not the necessity to break the walls and to do building works at home, there are no interferences with other devices (like in the radio communication case) or reflection problems (like in the infrared case), there is the possibility to put the modules in every place (it is sufficient to have an electrical socket in the nearness, or to use an extension cable), there is no need to have an extra power source (usually, in a bus cable domotic system, there is a direct voltage generated by a power supply and distribuited on the whole domotic net). 
Moreover, powerline communication (PLC, also called BPL in the USA, where the acronym stays for Broadband over Power Line, or NPL, Narroband over Power Line) is not only used in a home environment to create a virtual net among domotic modules, but is also used on the power distribution net to perform actions like reading the electricity meter, monitoring the power consumes and the state of a building, finding faults along the net, detecting illegal electricity usages and to solve the so-called last mile problem, that is the problem related to the final leg of delivering connectivity from a communications provider to a customer. In fact a cheap possibility to cover this final leg is using powerline communication.

Of course there are not only positive factors, but also some troublesome aspects that have to be considered. For example, if a noisy home appliance is connected to the powerline, it can disturb it and create problems over powerline communication (this can be easily solved using an appropriate powerline filter). Again, if a house is near an electric closet, the powerline may be unstable, with the same problems of the previous case.

The intent of this work is therefore to illustrate, going into more details, advantages and disadvantages of the powerline communication systems (PLCS), to show the differences between PLCS for power distribution net and PLCS for home and building environments, to indagate the methods to send data over the powerline, to explain which are the automations that is possible to connect and to control in a powerline domotic system and to show some case studies tackled by the authors.

\section{Advantages and disadvantages of power line communication}

Most private dwellings do not have dedicated neither low nor high-speed network cabling installed, and the labor costs required to install such wiring is often quite high. Power line communication is an emerging home networking technology that allows consumers to use their already existing electrical wiring systems to connect home appliances to each other and to the Internet (Dhir, A.\& Mousavi, S.,2001).

Home networks power-line technology can control anything that plugs into an outlet, including lights, televisions, thermostats, alarms, home automation modules and so on. If there is the availability of multiple power outlets in every room, the home power line infrastructure represents an excellent network to share data among intelligent devices, also with high data transfer rate, up to a few hundreds of Mbps (mega bit per second).

Unfortunately, there are some problems that have to be overcome and some aspects that have to be taken into account to realize a successfull data communication. They are listed in the following:

- minimum-security levels: powerlines do not necessarily provide a secure media

- data attenuation: due to the presence of numerous elements on a powerline network, data attenuation is likely to be an issue

- high costs of residential appliances: the cost of a powerline network modem is not always competitive with the cost of a standard modem used to connect to a phoneline network

- lack of global standards: there are several different standards for powerline communication, and the development of a global standard for distributing data over existing in-home powerline systems does not seem to be the trend of the international market

- noise: the greater amount of electrical noise on the line limits practical transmission speed (vacuum cleaners, light dimmers, kitchen appliances and drills are examples of noise sources that affect the performance of a powerline-based home network). 


\section{The noise problem}

Since the powerline distribution grid is present in almost every dwelling and building (on the contrary of other cabling, like xDSL - digital subscriber line - and similar), it represents a mean that offers a huge potential of communication services. Thus the electric power supply system is evolving from a pure energy distribution network to a multi-purpose medium, delivering energy, voice and other data communication services (Cuncic \& Bazant, 2003).

Unfortunately the power line channel has not been thought and designed to carry information other than electrical energy, so it has not good characteristics in terms of noise robustness and attenuation of high frequency signals. Due to this, power line represents a particularly difficult communication environment (Ferreira et al, 1996).

According to the measurements of Vines et al (1984), that are still valid nowadays, the sources of noise over the power line can be summarized as depicted in figure 1.

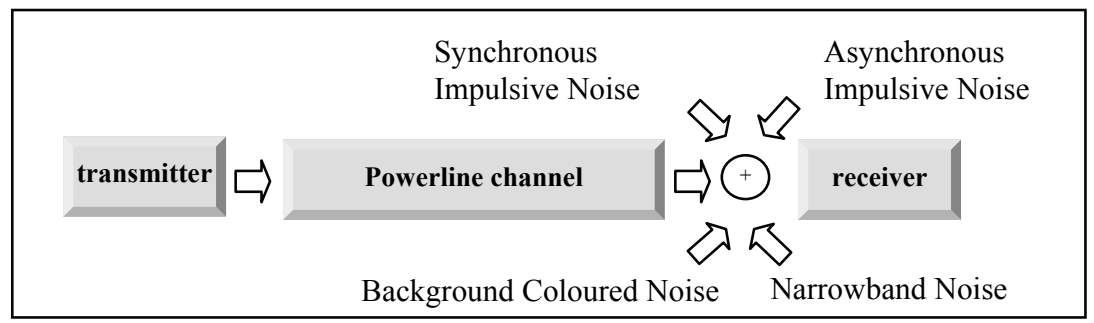

Fig. 1. Noise contributions over a power line network

- Background Coloured Noise shows a spectral power density that is low at medium frequency (around 20-30 MHz) but increases toward lower frequencies. It can be thought as the sum of many contributions, every one of each is a white noise acting in a non-overlapped frequency band. Its power spectral density (PSD) varies over time in terms of minutes or even hours (Zimmermann, 2002). This kind of noise can be caused by appliances like dimmers, hair dryers, computers and so on.

- Narrowband Noise is represented by portions of the spectrum with a very high level of PSD, but relegated in very thin and limited frequency ranges. In the frequencies beyond $150 \mathrm{kHz}$ it is mainly caused by amplitude modulated sinusoidal signals of broadcast radio stations in the medium and short wave bands $(1-22 \mathrm{MHz}$ typical), while up to approximately $150 \mathrm{kHz}$ it can be generated by switching power supplies, frequency converters, fluorescent lamps and television sets. The level is generally varying with daytime (Majumder \& Caffery, 2004).

- Synchronous Impulsive Noise is characterized by short voltage peaks, which are rare single events caused mainly by on and off switching actions, and which appear at frequencies that are multipliers of the main frequency ( $50 \mathrm{~Hz}$ for Europe, 60 for the USA). The pulses are of short duration (a few microseconds) and have a PSD decreasing with frequency. They are caused by rectifiers within DC power supplies and appliances with thiristor- or triac-based light dimmers.

- Asynchronous Impulsive Noise is said to be the worst kind of noise that afflicts powerline communications. In fact its time duration varies from few microseconds to milliseconds, and besides the uncertainty over this parameter, it is characterized by other three 
random variables: amplitude, pulse width and interarrival time (the time between two consequent noise pulses). The PSD of this noise can be grater than $50 \mathrm{~dB}$ above the background noise spectrum, and it can have contributions also up to $20 \mathrm{MHz}$. Due to its characteristics, this kind of noise can corrupt long blocks of data transmitted over the powerline. It is mainly caused by switching transients generated by switching power supplies.

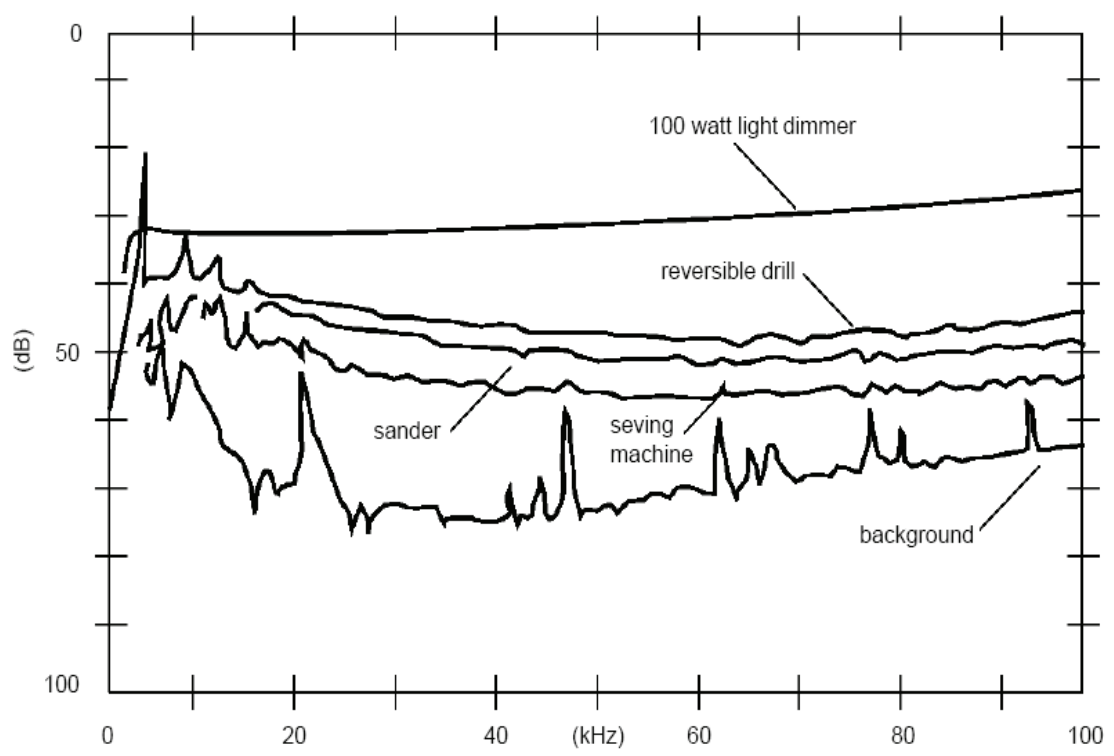

Fig. 2. Voltage spectra for three universal motors compared to light dimmers operating into the $60 \mathrm{~Hz}$ power circuit ( Vines et al, 1984)

From what mentioned above, it can be seen that the powerline is a communication mean difficult to be treated. Common analog modulation schemes like AM (amplitude modulation) or PM (phase modulation) are not so robust to be used in powerline communication systems, so other more complicated methods are exploited. They will be briefly illustrated in section 4 .

Besides being disturbed from several noise sources, the powerline itself creates some distrubances over other communication means. This is the case of the HF (high frequency) radio band, that can be upset by powerline systems in the range 1- $30 \mathrm{MHz}$. (Rhee et al 2008, Hansen 2002). Due to this phenomena and considering that powerline communications must not disturbe the powerline mean, that is already upset by its self, some regulations became necessary to manage the powerline communication field. This subject will be treated in section 5 .

\section{Modulation schemes}

Power line cables represent a very hostile environment, due to the fact that several different equipments connected to them can generate a wide variety of disturbances, causing the powerline to exhibit unpredictable and variable attenuation with time-variant and 
frequency selective behaviour. So transmitting data over them is not an easy task. Depending on the bit rate and on the frequency range used to injected data, the modulation methods are different.

Modulation is the teqnique that allows to send a signal (called modulating) by means of another signal, called carrier. The result of the interation between the two creates a new signal, called modulated, with different characteristics, more suitable to the behaviour of the transmitting channel (in this case the powerline). In general, the reason why the modulation is necessary is the low pass or band pass characteristic of the channel.

There are several modulation techniques. Besides the very simple and already mentioned analog modulation schemes, like AM, FM (frequency modulation) and PM, where the information resides respectively in the value of amplitude, frequency or phase of the modulated signal, for digital information, like in the case of PLC, other cathegories of modulation techniques are more appropriate: among them, the most simple are ASK (amplitude shift keying), where the information resides in the presence or absence of the modulated signal, FSK (frequency shift keying), where logic 0 and 1 are codified with two signals with different frequency, and PSK (Phase shift keying), where the information stays in signals with different phases.

All the mentioned modulation methods use a single and fixed carrier signal, and can be suitable for low trasmitting bit rates (up to a few hundreds of kbits/s) in domotic environments and also in rural telephony, to transmit voice with ranges of several hundred kilometers without repeaters.

Increasing the bit rate, noise is not the only problem, but also other disturbances have to be taken into account, for instance the intersymbol interference (ISI). This phenomena is due to the low pass behaviour of the powerline channel, which smooths the very ripid fronts of the digital information, like shown in figure 2.

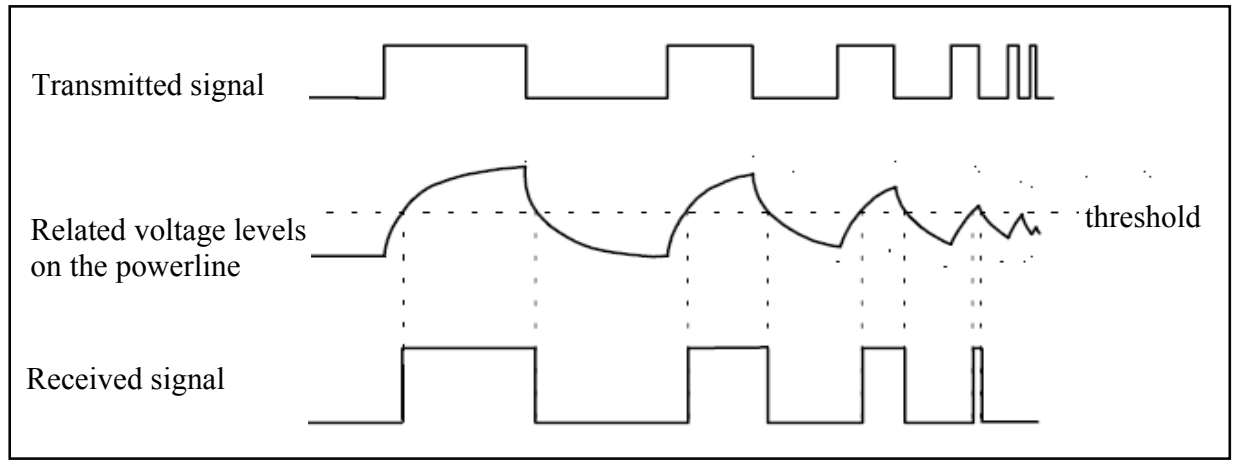

Fig. 3. The ISI problem

This is true for all kind of digital signals at every bit rate, but the difference between low and high bit rates is that if the delay caused by the low pass characteristic is greater than the symbol time duration and two consecutive bits are transmitted too close to each other, the receiver can be confused and can recognize a bit sequence different from the real one.

From what just mentioned, it is clear that ISI limits the maximum bit rate.

Several field tests have demonstrated that distributing information to separated carriers within the available bandwith is a successful way to lower the vulnerability against interference, attenuation and ISI problems. The most used modulation methods in this case 
are SST (spread spectrum techniques) and OFDM (orthogonal frequency division multiplexing).

Coming to an end, it can be stated that for low bit rates (up to some hundreds kbits/s) narroband modulation methods like PSK, FSK or also MSK (Minimum shift keying) and GMSK (Gaussian Minimum shift keying) can be used. For data rates around 1Mbits/s the CDMA (Code-division multiple access) technique may provide an effective solution, while for high-speed services, with data rates of dozens or hundred of Mbits/s, only techniques like SST, resistant to all kind of narrowband interference, or OFDM, resistant to frequency selective noise, are suitable.

In any case, to increase the robustness of the communication link, besides the adopted modulation technique there are also other digital messages manipolations methods, like ARQ (Automatic Repeat reQuest), FEC (Forward Error Correction) and CRC (Cyclic Redundancy Check).

Interesting dissertations about modulation schemes and error detection methods are illustrated in Karl \& Dostert (1996), Waldeck \& Dostert (1996), Han Vinck at al (2000), Lin et al (2002), Cuncic \& Bazant (2003), Hakki Cavdar (2004).

\section{Regulations}

Concerning regulations, since PLC devices are electric equipments that transmit over the power line (being both in the electric and in the telecommunication field), powerline networks, products and services have to work under regulations regarding (Napolitano, 2004):

- $\quad$ electric safety

- electromagnetic compatibility

- $\quad$ nets and communication services.

Different regions of the world have different kind of directives and tolerated levels.

In the European Community, regarding the first point, everything connected to the powerline cables must respect the low voltage directives, that in Europe are Regulations 73/23/EEC, 93/68/EEC and EN 60950 (Safety of information technology equipment - ITE- also regarding devices destinated to use the power line network as a transmission mean).

Regarding the third point, the European Regulations are:

- Directive 2002/19/EC on access to, and interconnection of, electronic communications networks and associated facilities (Access Directive)

- Directive 2002/20/EC on the authorization of electronic communications networks and services (Authorization Directive)

- Directive 2002/21/EC on a common regulatory framework for electronic communications networks and services (Framework Directive)

- Directive 2002/22/EC on universal service and users' rights relating to electronic communication networks and services (Universal Service Directive).

Regarding the second point, we have to distinguish between PLC used at low frequencies to send small amount of data (like in the case of home and building automation) and PLC used to realize an internet connection, used at high frequency to share big amount of data.

In the first case (Lauder\& Sun, 1999) regulation is EN 50065-1 (in the band 3-148.5 kHz), in the second one is EN 55022, that specifies limits for conducted disturbance at the mains ports in the frequency range $150 \mathrm{kHz}-30 \mathrm{MHz}$. 
Outside the Region 1 of the International Telecommunications Union (ITU), which covers Europe, there is no Long Wave broadcast band and the applicable standard for mains-borne communications systems is IEC 61000-3-8 (regarding Electromagnetic compatibility). This standard permits significant levels of signal injection up to $525 \mathrm{kHz}$, just below the start of the Medium Wave broadcast band.

Regarding the immunity, and not the radiated emissions, CENELEC EN 50412-1:2004 (Immunity requirements for power line communication apparatus and systems used in lowvoltage installations in the frequency range $1,6 \mathrm{MHz}$ to $30 \mathrm{MHz}$-- Part 1: Residential, commercial and industrial environment) and EN 55024 (Information technology equipment - Immunity characteristics - Limits and methods of measurement) are reference regulations.

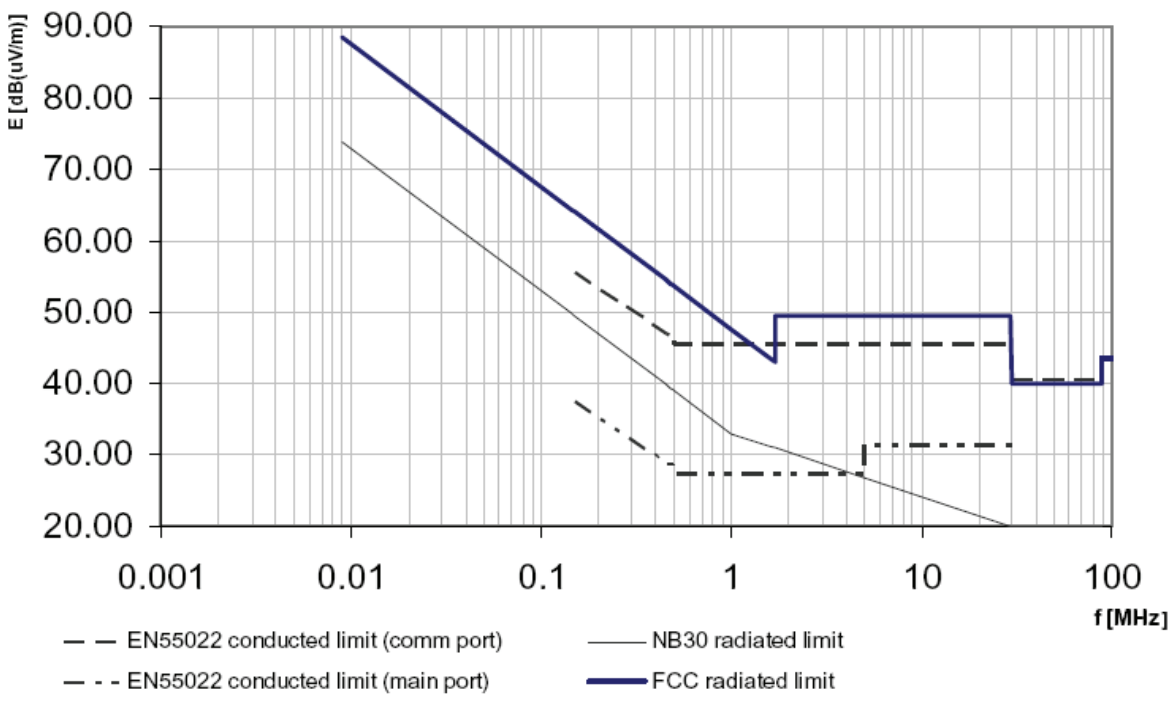

Measurement at $3 \mathrm{~m}$ and Quasi-peak detector

Conversion factor between conducted and radiated levels is $-28.5 \mathrm{~dB}$

Fig. 4. Regulation limits for PLC conducted and radiated emissions

The case of home and building automation falls within the scope of the EN 50065-1 regulation, which specifies (Cenelec, 2002), that the sub band 3-95 kHz is limited to energy providers and the sub band $95-148,5 \mathrm{kHz}$ is destinated to user applications. In this last band the equipments are said to be Class 122 or Class 134 (in the old version of the EN50065-1, Class 122 was Class 116). The regulation sais that every PLC device must use the frequency of $132,5 \mathrm{kHz}$ to inform that it wants to start a transmission and in the band $125 \mathrm{kHz}-140 \mathrm{kHz}$ a CSMA (Carrier Sense Multiple Access) access protocol is mandatory.

In the USA regulations for radiated limits are enacted by FCC (Federal Communications Commission). They are more permissive and allow plc modem to attain higher data rate than the European Standards (Schneider et al, 2004).

The German Regulatory Authority for Telecommunications and Posts (called Reg TP) established the NB30 limits which are based on radiated measurements. Contrary to the previously discussed standards, the limits are given in peak levels. It is difficult to make a direct comparison with other standards since the conversion factor depends on the measurement setup. 
United Kingdom in the past used the MPT1570 standard, that specified limits up to $30 \mathrm{MHz}$ until January 2003. The current version specifies radiated limits below 1.6 MHz only. PLC modems do not need to be compliant with this standard if they operate in another frequency band.

Recently also the IEEE Society (Institute of Electrical and Electronic Engineers) has shown some interest in standardization of the PLC field, so a new commettee (TC-PLC: Technical Committee on Power Line Communications ) is born, sponsorising two standards:

- IEEE P1901: Standard for Broadband over Power Line Networks: Medium Access Control and Physical Layer Specifications

- IEEE P1775: Powerline Communication Equipment - Electromagnetic Compatibility (EMC) Requirements - Testing and Measurement Methods.

The areas of interest of the Technical Committee are the analytical, theoretical, simulation, experimental, and practical aspects of digital communications over power lines.

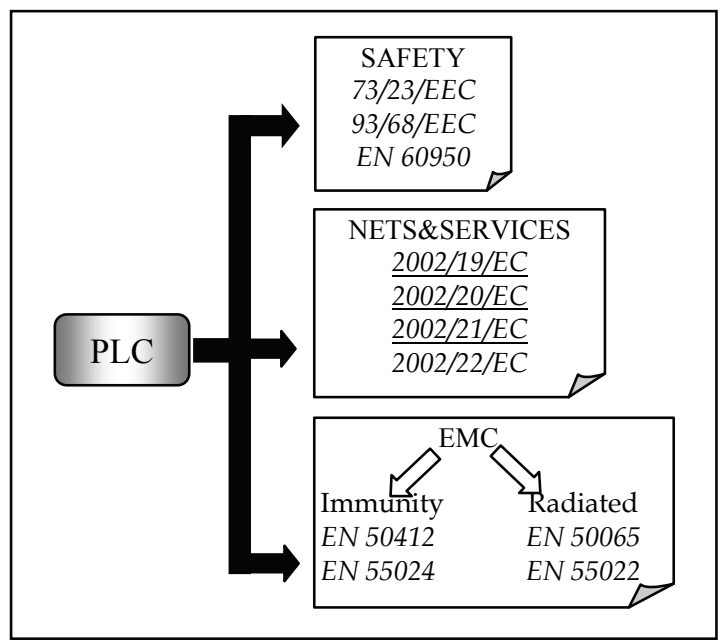

Fig. 5. PLC European regulations

\section{Powerline applications}

Powerline communication systems can be classified into four distinct cathegories, concerning the type of services that they can provide: communication over high voltage grid, access to an internet provider, in-home networking with high data rate and in-home simple control application with low bit rate.

Regarding the first cathegory, the available services for electricity provider companies, as mentioned in the first section of this paper, concern reading the electricity meter, monitoring the power consumes and the state of a building, finding faults along the net, detecting illegal electricity usages (Hakki Cavdar $b$, 2004), trasmitting telephone signals in rural regions.

Access powerline technologies send data over the low-voltage electric network that connects the consumer's home to the electric utility provider. The powerline access technologies enable a last mile local loop solution that provides individual homes with broadband connectivity to the Internet. 
In-home powerline technology communicates data exclusively within the consumer's dwelling and extends the connectivity to all the electrical outlets within the home. The same electrical outlets that provide power will also serve as access points for the network devices. Whereas the access technologies focus on delivering a long-distance solution, competing with $\times$ DSL and broadband cable technologies, the in-home powerline technology focuses on delivering a short-distance solution.

Services like powerline intranet solutions and powerline music distribution (that have to follow the EN 55022 directive) belong to the in-home networking cathegory. Their typical bit rate can achieve values of $200 \mathrm{Mbps}$ (declared). These kind of services compete against other in-home interconnection technologies such as wireless, even if up to now the bit rates are definitely lower (Lin, Y et al, 2003).

Regarding the last cathegory, it can provide services like sending small amount of data with low bit rate (in fact to open an automatic door or to control the switching on and off of a light not big amount of data are required). Belong to this cathegory the services of the home and building automation. They have to follow the EN 50065 directive.

\section{Home-Building automation and powerline}

Many, but not all, Home and Building Automation system producers sell modules that can communicate over the powerline.

A few examples are mentioned in the following.

Konnex, the European consortium that was born from the join of Batibus, EHS (European Home System) and EIB (European Installation Bus), has defined a standard that up to now covers three physical mediums. They are Twisted Pair (called TP0 and TP1 variants with data rates of 1200 and 2400 baud), Radio frequency (RF with $868 \mathrm{MHz}$ carrier and data rate 16.384 baud) and Power line (called PL110 specification with data rate of 1200 baud and PL132 variant with center frequency of $132.5 \mathrm{kHz}$, FSK modulation with CSMA/CA, FEC, 16 bit CRC and data rate equal to 2400 baud).

X10 is an open standard very widespread in the USA and in Europe. It uses an ASK modulation, synchronized with the zero crossing of the power line signal $(110 \mathrm{~V}, 60 \mathrm{~Hz}$ in the USA and $220 \mathrm{~V}, 50 \mathrm{~Hz}$ in Europe). It has an effective bit rate of $60 \mathrm{bps}$ and it does not implement FEC or CRC techniques. A binary 1 is represented by a 1 millisecond burst of $120 \mathrm{kHz}$ at the zero crossing point, and a binary 0 by the absence of the $120 \mathrm{kHz}$ signal.

CEBus is an open standard proposed by the Electronic Industries Association (EIA). It defines a set of rules for consumer products to communicate with each other (Dhir, A.\& Mousavi, S., 2001). The CEBus base products consist of two fundamental components, a transceiver and a microcontroller. Data packets are transmitted by the transceiver at about $10 \mathrm{Kbps}$. The CEBus standard includes commands such as volume up, fast forward, rewind, pause, skip, and temperature up or down one degree. These commands are based on a language called Common Application Language (CAL). CEBus uses spread spectrum technology to overcome communication impediments found within the home's electrical powerline. The CEBus powerline carrier spreads its signal over a range from $100 \mathrm{~Hz}$ to 400 $\mathrm{Hz}$ during each bit in the packet.

To avoid data collisions, CEBus uses a Carrier Sense Multiple Access/Collision Detection and Resolution (CSMA/CDCR) protocol.

A CEBus-based home network is comprised of a control channel and potentially multiple data channels on each of the CEBus media. CEBus control channel is used exclusively to 
control devices and resources of the network, including data channel allocations. Data channels typically provide selectable bandwidths that can support high data rates and are used to send data such as audio, video, or computer files over the network. The characteristics of a data channel can vary greatly depending upon the medium and connected device requirements. All data channel assignments and functions are managed by CEBus control messages sent via the control channel.

LONWorks technology is a solution for control networks developed by Echelon Corporation. It has been estimated that more than six million LONWork based appliances are installed worldwide. A LONWorks system includes all the necessary hardware and software components for implementing complete end-to-end control systems. In a LONWorks network, intelligent control devices, called nodes, communicate with one another using a common protocol. Each node in the network contains embedded intelligence that implements the protocol and performs control functions. In addition, each node includes a physical interface (transceiver) that couples the node microcontroller with the communications medium. A typical node in a LONWorks control network performs a simple task. Devices such as proximity sensors, switches, motion detectors and sprinkler systems may all be nodes on a home network. The core of a node is the Neuron chip, which is basically a microcontroller specifically designed to offer a good cost-effective solution available for home control devices. Appliances on a LONWork enabled home network use a protocol to communicate with each other. This protocol is known as LonTalk and has been approved as an open industry standard by the American National Standards Institute (ANSI) - EIA 709.1.

The LONWorks Network Services (LNS) architecture provides a range of network services to appliances that are connected to a control system. A couple of examples of Lonworks powerline modem are PL3120 and PL3150. They work under EN 50065 regulation, using a BPSK (binary phase shift keying) modulation, a primary frequency of $132 \mathrm{kHz}$ in band C $(125-140 \mathrm{kHz})$ of the EN 50065 and of $86 \mathrm{kHz}$ in band A $(40-90 \mathrm{kHz})$. If these primary frequencies do not work properly, they can switch over a secondary frequency $(115 \mathrm{kHz}$ in banc $\mathrm{C}$ and $75 \mathrm{kHz}$ in band $\mathrm{A}$ ).

Another important player in the PLC world is the Home Plug Alliance, formed by very important industry leaders such as Intel, LG, Cisco Systems, Motorola, Samsung, Sharp, Texas Instruments and others. The Alliance created three Promoters' Groups focused on specific standards initiatives within the Alliance. Initially, the Promoters' Groups will address three key technology areas:

- HomePlug 1.0 + AV (in-home connectivity, including digital home and consumer electronics applications)

- HomePlug BPL (to-the-home, Broadband-over-Powerline applications)

- HomePlug Home Automation (command-and-control applications)

The alliance's effort to develop a specification for an advanced command and control technology for home automation, known as HPCC (HomePlug Command \& Control) is well underway. Command and Control is a low-speed, very low-cost technology intended to complement the alliance's higher-speed powerline communications technologies, which is specified in the Homeplug 1.0 standard and is not intendend for home automation purposes but for home networking, allowing multiple home desktop and notebook computers to be networked to share an Internet connection, printers, files, to play games and so on. OFDM is the basic transmission technique used by the HomePlug 1.0. Cyclic prefix and differential 
modulation techniques (DBPSK, DQPSK) are used to completely eliminate the need for any equalization. Impulsive noise events are overcome by means of forward error correction (FEC) and data interleaving. Privacy is established by creating logical networks through the use of encryption. HomePlug's privacy and security scheme is based on the 56-bit Data Encryption Standard (56-bit DES). The maximum baud rates depends on the level they are considered. The physical layer can achieve a rate of $13.78 \mathrm{Mbps}$, and the MAC (Medium Access Control ) layer 8.2 Mbps.

\section{Experiments and case studies}

The interest of our research group is focused on the field of low cost home automation systems, mainly devoted to disabled people.

First of all, at the beginning of our interest in this field, we tried to explore applications regarding the implementation of powerline node by our selves.

This has been done both designing the interface electronics with the powerline and using consumer off the shelf powerline modems with a TTL (Transistor Transistor Logic) interface for our embedded hardware.

Regarding the first case, at the beginning we developed a simple two points communication link with an ASK modulation with a frequency of $75 \mathrm{KHz}$, and after that a more robust communication link with a BFSK modulation, using the ST7540 power line transceiver produced by ST Microelectronics.

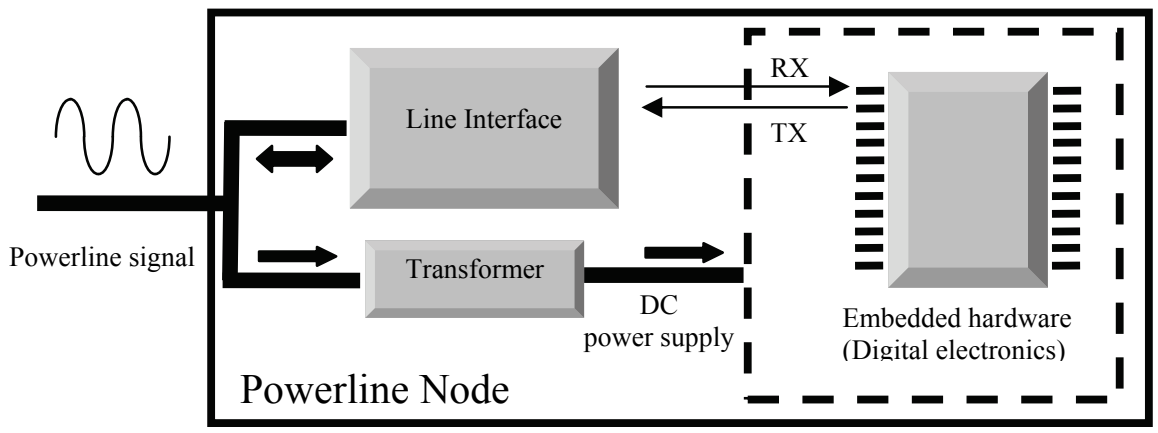

Fig. 6. General structure of a powerline node

A few tests had been made on these simple electronics, revealing that the performances of the ASK link in terms of noise robustness were considerably under the ones of a commercial power line modem, instead the link built using the ST 7540 was robust and affordable.

In any case, whatever may be the powerline node hardware, the general structure is depicted in figure 6 . The node needs some electronic components (line interface) to be coupled to the powerline. The line interface is typically formed by an isolation transformer and some capacitors and inductances to implement selective filters. The DC power supply for the digital section of the node can also be obtained from the powerline voltage, typically by means of a transformer and a rectifier. The embedded hardware is generally a digital microcontroller that manages the transmission protocol and executes or superintends to its own particular domotic function (controlling a light, opening a door or a window and so on).

After this practical trials our efforts have been focused on a higher level. 
Regarding the second case, we implemented a communication link between two FSK commercial powerline modems (Mainardi et al, 2005) with the usage of the SNAP (Scaleable Node Address Protocol) protocol (SNAP, 2002). The final demonstrative application was the opening and closing of a window in an environment designed for disabled people (the Centro $\mathrm{H}$, a centre for information about disability in the city of Ferrara).

The reason why we decided to use this protocol reside in the fact that it is a scalable protocol, depending on the user's requests, it is very light regarding the necessary CPU resources, it can addresses 16,7 milion nodes, it can support 8 different check error methods and also broadcast messages, it is mean independent (it does not matter if one uses a powerline or an RF link) and can be implemented both for synchronous and asynchronous communication channels.

The main achieved goal of this first step was to demonstrate that is possible to implement a very cheap powerline application using off the shelf hardware and a free protocol, without any license cost.

The aim of this work was to demonstrate that is not necessary to spend a lot of money to build a home automation system, and that is possible to leave behind the troubles on choosing among a lot of possible commercial solutions using very few, simple, cheep and off the shelf components.

The used powerline modem was a PLM-01, produced by High Tech Horizon, with a baud rate of 2400 bps, which utilized a ST7535 integrated circuit (IC), with a frequency of 131.85 $\mathrm{kHz}$ to transmit a logic 1 and $133.05 \mathrm{kHz}$ to transmit a logic 0 .

After these two simple experiments, we decided to test a commercial powerline system, to verify its potentialities.

Our choice fell on the Marmitek X10 powerline system.

$\mathrm{X} 10$ is a standard and open protocol for powerline communications, that allows to address up to 256 devices (enough for a standard apartment). Every address code is formed by a number between 1 and 16 and a letter between A and P (for instance A3 or P14 are valide codes). The used modulation technique is an ASK, and the frequency of the signal is $120 \mathrm{kHz}$. With this kind of system we equipped a small flat of about $80 \mathrm{mq}$ (Mainardi \& Banzi 2007), owned by the already mentioned Centro $H$, with automations for doors, windows, rolling shutters and lights. The advantages of a X10 system concern the fact that it uses an open protocol, it does not require connecting other cables, respect to those already present for the standard power line, is very cheap, it can be used with a low cost just to implement basic domotic functions (often disabled people do not need the entire house to be automatized, but is sufficient for them to have a few rooms, typically the bed room, the bath room and the dining room, with a minimum level of technology and an adequate cost), it can be easily extended to more sophisticated functions, is provided by a lot of electronic firms, with dozens of different modules, is very easy to use, also for inexperienced (unskilled) people, it can be easily used to implement experimental devices in the educational field.

After having installed this system in the apartment and having verified its excellent and stable functioning for more than two months, we decided to develop a user friendly interface for disabled people (Mainardi, 2008). Another key point of domotics concerns in fact the control interface (Thornett \& Brown, 1988, Vaughan et al, 2006). Domotic systems 
are usually controlled by means of switches, in-wall touchscreens, remote controllers or PDAs. These interfaces are useful for people with no health problems, no handicaps, no restrictions in mobility, but creates many difficulties for people that for example have low vision, or hand trembling, people with severe spinal cord injuries and so on. It is true that there are a few dedicated devices for disabled people to control domotic systems with alternative inputs like the voice (Sicare Pilote and Easy by Voice System are two examples), but they are still expensive and represent a very small part of the market, with a consequent little possibility of choice, or they are still research projects, not available on the market. Besides this, there are many disabled that cannot use the voice, but for instance they can move a little part of their body.

In other words, domotic systems and products are thought mainly for able-bodied people, with attentions to many expensive details of aesthetics and ergonomics, and only at a second time for disabled people, that maybe can take more advantages of them in terms of autonomy and indipendence. So the project was focused on the design and the realization of a portable touchscreen interface for disabled people, used to control a low cost powerline domotic system. The interface has been implemented for Marmitek modules, but of course, knowing the infrared protocol of other domotic systems, it will be possible to make the device working also with other products, simply adding a software routine.

This interface has a touchscreen input, with icons big and separated enough for people with low vision or hand trembling (in any case the icons, their shape, size and subject, were approved by personnel and disabled users of the Centro $\mathrm{H}$ ). The icons are organized in a three level menu, like shown in figure 7: the first general menu has an icon for every of the six rooms (the kitchen, the dining room, the bathroom, the corridor and two bedrooms). Touching on a room image the second menu will open, with an icon for every automation of that room (light, window, door or rollingshutter). Touching again the third menu will appear, with the options for every automation (turn on/off the light, rise/fall the rolling shutter and so on).

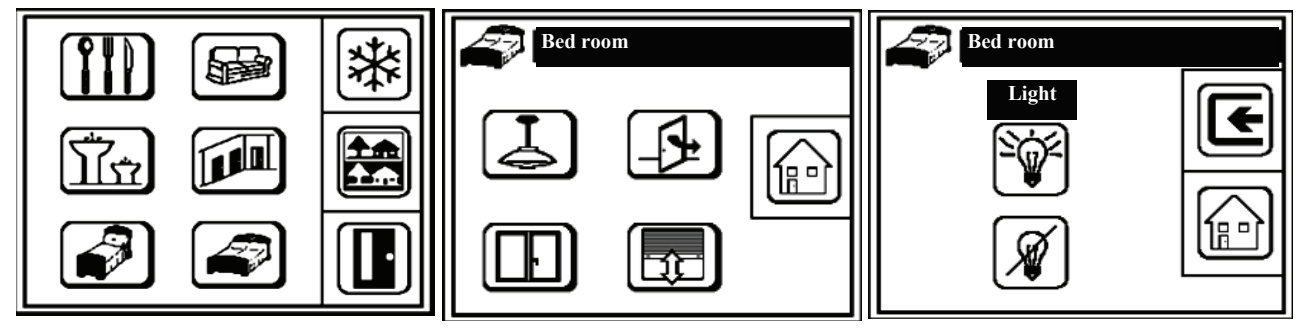

Fig. 7. Three levels of the portable touchscreen interface menu

The general structure of the interface is shown in figure 8 .

The interface communicates with the Marmitek powerline system by means of an IR (infrared) link, sending IR messages to the Marmitek IR7243 device, that translates them into X10 commands over the powerline. The modules used to control the automations were the Marmitek UM7206, AWM2 and LW12, while to control scenarios (sets of X10 commands) a CM11 module was utilized, as shown in figure 9. More details of this implementation can be found in Mainardi, 2008. 


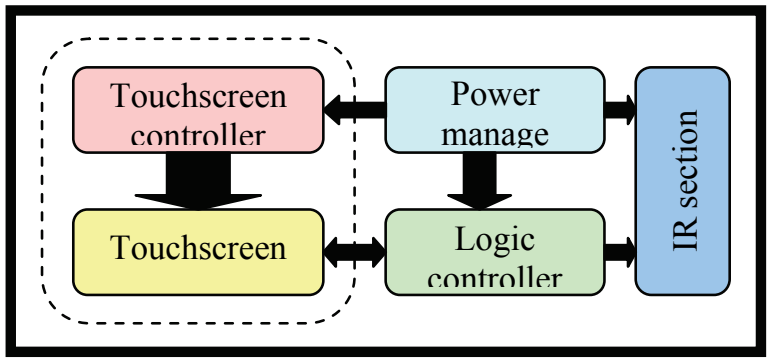

Fig. 8. Portable touchscreen interface structure

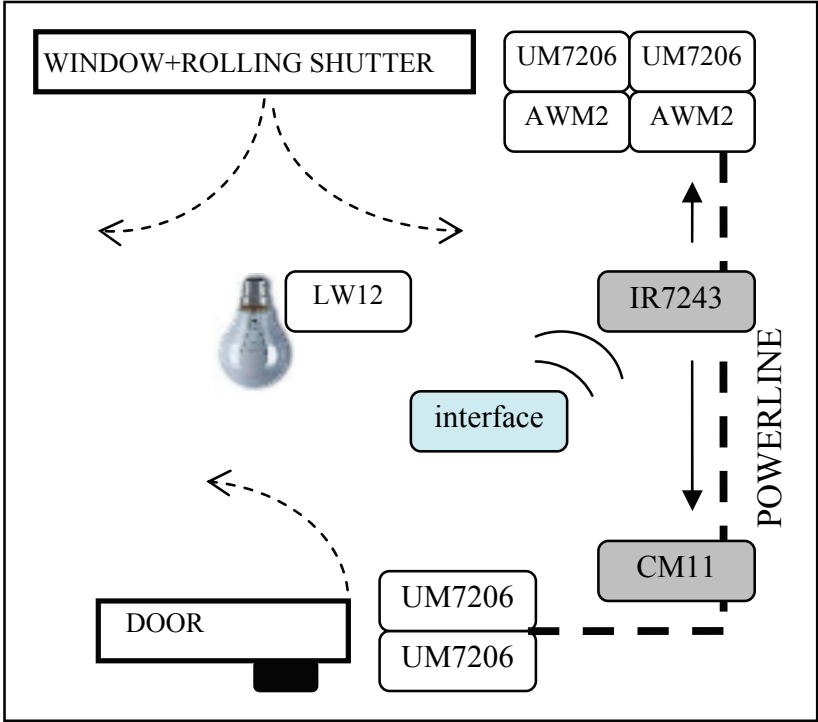

Fig. 9. Example of a room controlled with the Marmitek X10 domotic system

Another interesting Marmitek module is the XM10E, that is an interface between the X10 codes for powerline and the TTL electronic world. Using this device is possible to develop several applications, if they are not available in the wide X10 world. Using the XM10E module, everyone can create a simple hardware with a microcontroller that reads and sends every X10 code over the powerline, being able to control every possible automation connected to a Marmitek powerline module.

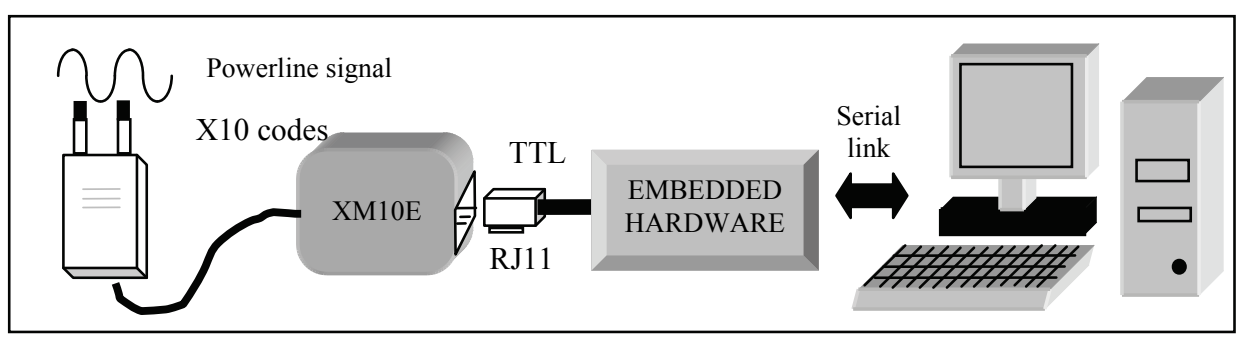

Fig. 10. Creating X10 applications with the XM10E module 
After having design an embedded TTL electronics able to communicate with the XM10E, the further step has been creating a serial link with a computer, so that by means of a graphical interface every user can manage the whole domotic X10 system. In fact the user can write a code and a command using the keyboard of the PC, (for instance: turn on the ligth managed by the $\mathrm{X} 10$ modules with A1 address), this command is sent by means of the serial link to the embedded hardware, that converts it following a certain protocol and communicates a $\mathrm{X} 10$ code to the $\mathrm{XM10E}$, that translates it over the powerline, as shown in figure 10.

Anyway, independently from the described application, the XM10E allows the implementation of every desired function or device, and for this reason is a tool that provides excellent flexibility to the Marmitek system.

\section{How to set up an environment for a domotic powerline system}

To arrange a home environment for a conventional home automation system, cabled with a standard bus cable, is necessary to create separated electrical tubings for the bus or to think about larger conventional electrical tubings, able to carry both the powerline cables and the bus cable and totally interconnected from each other. Is necessary also to take the bus everywhere in the home environment (also near the windows and the doors, considering the possibility to motorize them) and to arrange a power supply (or more than one, if the bus line is longer than a fixed length), typically with a so called SELV voltage (Safety Extra Low Voltage ) of about $27-29 \mathrm{~V}$. If the same home automation system is realized with the powerline technique, the electric plant has not to be modified. The electrical tubings can have the standard diameter and no other electrical traces have to be installed. The only essential things are taking the powerline cables near doors and windows, arranging several outlets in every room, at least three or four, and being sure that all the sockets are served by the same powerline chain, without thermal magnetic or differential switches among them, because these devices, filtering the line, block every signal potentially overlapped to the powerline.

\section{Powerline as a successful educational tool}

Powerline world is definitely an interdisciplinary field, that involves electronics, telecommuncation, informatics and automation. Thus, from an academical point of view, is an excellent tool in the educational effort for engineering students.

Regarding our experience, several students of the Automation Group of the University of Ferrara took their degree with a graduation thesis based on projects concerning powerline and domotics applications. A few students designed the interface electronics with the powerline mean, a few studied the powerline communications, others designed X10 systems, others again designed customer electronics to develop new tools for the domotic X10 system. These activities are very useful in the sense that the students can touch with their own hands the real working of what they study in theory, also cooperating with the final users of this domotic systems, that in our case are disabled people that live and work inside the apartments, kindly placed at our disposal from the Centro $\mathrm{H}$.

\section{Conclusion}

Power line communication is a valid technique that allows the exchange of data by means of the powerline cables that are present in every dwelling and in every building. Even if there 
are some problems of noise and possible interferences, modern modulation methods make PLC systems in general affordable and reliable. Information transmitted through the powerline can be used to share data with high bit rates (generally used for home intranets, for the last mile problem or for in-home music distribution), or also to control home and building automation systems, that typically need lower bit rates, up to a few kbps (kilo bit per second).

Several home system producers, like Lonworks and Konnex, besides the standard devices that communicate by means of a bus cable, sell powerline modules, but the most cheapest solution for a powerline system is represented by the X10 standard, that allows to control small domestic environments. Setting some basic functions in a X10 system is very easy. There are 256 available addresses, that one can set directly on the module, simply with a screwdriver. Two minutes are enough to control a light with an IR or a RF remote controller. We can't say the same for the other systems, where an expert is necessary to install and program (via software or directly on the back of the equipments) the modules and their functions.

Equipping a home environment with a smart powerline domotic system, beside its possibilities of energy saving and comfort increasing, is also one of the way to try to give back a certain level of autonomy to people with special needs. A smart home system can improve their independence in the every day's activities, in a comfortable environment which is very personal and peculiar for everyone, in any case different from a hospital-like setting.

\section{Future research}

After having tried with several practical X10 experiments, further steps will be explore other powerline systems (like Lonwork or Konnex) to test their potentialities, also conducting a comparative study on the power consumes of home and building automation systems.

Another key point remains in any case the world of domotic control interfaces for disabled people, so our effforts will be again concentrated on this topic.

\section{References}

Cenelec (Comitè Européen de Normalisation Electrotechnique), (2002). EN 50065-1 Signalling on low-voltage electrical installations in the frequency range $3 \mathrm{KHz}$ to 148,5 kHz - Part 1: General requirements, frequency bands and electromagnetic disturbances, July 2007

Cuncic, P. \& Bazant, A. (2003). Analysis of Modulation Methods for Data Communications over the LOW-voltage Grid, Proceedings of $7^{\text {th }}$ International Conference on Telecommunications, pp.643-648, ISBN 953-184-052-0, Croatia, June 2003, Zagreb

Dhir, A.\& Mousavi, S. (2001). Home Networking Using “No New Wires” Phoneline and Powerline Interconnection Technologies, available at http://www.xilinx.com/ support/ documentation/white_papers/wp133.pdf

Ferreira, H.C.; Grovè, H. M.; Hooijen, O. \& Vinck, A.J. (1996). Powerline Communications: an Overview, Proceedings of $4^{\text {th }}$ AFRICON Conference, Volume 2, pp. $558-563$, 24-27 Sept. 1996

Gelman, A. (2006). Communications Society and Standards, Proceedings of ICC 2006 (International Conference on Communications), 11-15 June 2006, Istanbul, TURKEY 
Gotz, M.; Rapp, M. \& Dostert. K. (2004). Power Line Channel Characteristics and Their Effect on Communication System Design, IEEE Communications Magazine, April 2004, pp. 78-86

Hakki Cavdar. (2004), Performance analysis of FSK power line communication systems over the time-varying channels: measurements and modeling, IEEE Transaction on Power Delivery, VOL. 19, No.1, January 2004, pp. 111-117

Hakki Cavdar, I. (2004), A Solution To Remote Detection Of Illegal Electricity Usage Via Power Line Communications, IEEE Transactions On Power Delivery, Vol. 19, No. 4, October 2004

Hansen, D. (2002). Update on Power Line Telecommunication Activities in Europe, IEEE International Symposiumon Electromagnetic Compatibility, 19-23 Aug.2002 , Vol.1, pp:17-22, Minneapolis, USA, ISBN: 0-7803-7264-6

Han Vinck, A. J.; Haering, J. \& Wadayama, T. (2000). Coded M-FSK for Power Line Communications, Proceedings of ISIT 2000 (IEEE International Symposium on Information Theory), pp. 137, June 2000, Sorrento, Italy

Karl, M. \& Dostert, K. (1996). Selection of optimal modulation scheme for digital communications over low voltage power lines, Proceedings of IEEE 4th International Symposium on Spread Spectrum Techniques and Applications, pp. 1087-1091, Vol. 3, September 1996

Lauder, D. \& Sun, I. (1999). Modelling and Measurement of Radiated Emission Characteristics of Power Line Communications Systems for Standards Development, Proceedings of IEEE International Symposium on Power Line Communications and Its Applications, Lancaster UK, 30 March - 1 April 1999

Lin, Y.J.; Latchman, H. A. \& Lee, M. (2002). A power line communication network infrastructure for the smart home, IEEE Wireless Communications, December 2002, Vol.9, pp:104-111, ISSN: 1536-1284

Lin, Y.; Latchman, A. \& Newman, R. E. (2003). A comparative performance study of wireless and power line networks, IEEE Communications Magazine, April 2003, Volume 41, Issue 4, pp: 54 - 63

Mainardi, E.; Banzi, S.; Bonfè, M. \& Beghelli, S. (2005) A low-cost Home Automation System based on Power-Line Communication Links, Proceeding of ISARC 2005 (International Symposium on Automation and Robotics in Construction), September 2005, Ferrara (Italy)

Mainardi, E. \& Banzi, S. (2007). The Centro-H smart homes, a point of convergence between technology and social sciences, Proceedings of AAATE 2007, pp:96-100, 3-5 October 2007, San Sebastian, Spain

Mainardi, E. (2008). Design of a portable touchscreen interface for powerline domotic systems, accepted for CASE 2008 (Conference on Automation, Science and Engineering), August 2008, Washington (USA)

Majumder, A. \& Caffery, J. (2004). Powerline Communications: an overview, IEEE Potentials, Vol. 23, Issue 4, Oct.-Nov. 2004, pp: 4 - 8

Napolitano R. (2004). PowerLine Telecommunications: National and International Regulamentations, available at http://www.aei.it/ ita/federAEIT/ PLC_01_12_2004 / 11.Napolitano.pdf, Milan 01.12.2004 (in Italian)

Rhee, J.; Rhee, E. \& Park, J. (2008); Electromagnetic interferences caused by power line communications in the HF bands, Proceedings of IEEE International Symposium on 
Power Line Communications and Its Applications (ISPLC), 4 April 2008, pp. 249 - 252, Korea

Schneider, M.; Favre, P. \& Rubinstein, M. (2004). Emc Analysis Of Powerline Systems, Final Report of IEEE International Symposium on Power Line Communications and Its Applications, July 2004

S.N.A.P. Scaleable Node Address Protocol, (c) 1998-2002 HTH, Document revision 1.03, Document revision 1.03, (C) 1998-2002 HTH, Document revision 1.03

Sun, M.C. \& Lun, D.P.K. (2002). Power-line communications using DWMT modulation, Proceedings of the IEEE International Symposium on Circuits and Systems, ISCAS 2002, Vol.4, pp:493-496, ISBN: 0-7803-7448-7

Thornett, C. E. \& Brown,. A. W. (1988). Interfacing modern technology to disabled patients, IEEE Colloquium on Engineering Design for the Disabled, 10 Nov 1988, pp:9/1 - 9/2

Vaughan, T.; McFarland, D. J.; Schalk, G.; Sarnacki, W.A.; Krusienski, D.J.; Sellers,E.W. \& Wolpaw, J.R. (2006). The wadsworth BCI research and development program: at home with BCI, IEEE Transactions on Neural Systems and Rehabilitation Engineering, Volume 14, Issue 2, June 2006 pp:229 - 233

Vines, R.M.; Trussell, H.J.; Gale, L.J. \& Ben O'neal, J. (1984). Noise on Residential Power Distribution Circuits, IEEE Transactions on Electromagnetic Compatibility, Volume EMC-26, Issue4, Nov.1984, pp:161-168

Waldeck, T. \& Dostert, K. (1996). Comparison of modulation schemes with frequency agility for application in power line communication systems, Proceedings of IEEE 4th International Symposium on Spread Spectrum Techniques and Applications, pp. 821825,Vol . 2, Issue , 22-25 September 1996

Zimmermann, M. \& Dostert, K. (2002). Analysis and modeling of impulsive noise in broadband powerline communications, IEEE Transactions on Electromagnetic Compatibility, Vol . 44, Issue1, Feb.2002, pp:249-258 


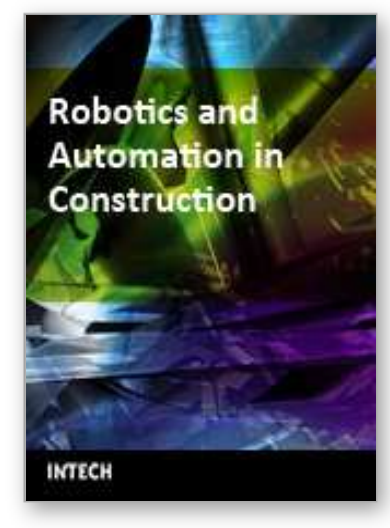

\author{
Robotics and Automation in Construction \\ Edited by Carlos Balaguer and Mohamed Abderrahim
}

ISBN 978-953-7619-13-8

Hard cover, 404 pages

Publisher InTech

Published online 01, October, 2008

Published in print edition October, 2008

This book addresses several issues related to the introduction of automaton and robotics in the construction industry in a collection of 23 chapters. The chapters are grouped in 3 main sections according to the theme or the type of technology they treat. Section I is dedicated to describe and analyse the main research challenges of Robotics and Automation in Construction (RAC). The second section consists of 12 chapters and is dedicated to the technologies and new developments employed to automate processes in the construction industry. Among these we have examples of ICT technologies used for purposes such as construction visualisation systems, added value management systems, construction materials and elements tracking using multiple IDs devices. This section also deals with Sensorial Systems and software used in the construction to improve the performances of machines such as cranes, and in improving Human-Machine Interfaces (MMI). Authors adopted Mixed and Augmented Reality in the MMI to ease the construction operations. Section III is dedicated to describe case studies of RAC and comprises 8 chapters. Among the eight chapters the section presents a robotic excavator and a semi-automated façade cleaning system. The section also presents work dedicated to enhancing the force of the workers in construction through the use of Robotic-powered exoskeletons and body joint-adapted assistive units, which allow the handling of greater loads.

\title{
How to reference
}

In order to correctly reference this scholarly work, feel free to copy and paste the following:

Elena Mainardi and Marcello Bonfe (2008). Powerline Communication in Home-Building Automation Systems, Robotics and Automation in Construction, Carlos Balaguer and Mohamed Abderrahim (Ed.), ISBN: 978-9537619-13-8, InTech, Available from:

http://www.intechopen.com/books/robotics_and_automation_in_construction/powerline_communication_in_ho me-building_automation_systems

\section{INTECH}

open science | open minds

\author{
InTech Europe \\ University Campus STeP Ri \\ Slavka Krautzeka 83/A \\ 51000 Rijeka, Croatia \\ Phone: +385 (51) 770447 \\ Fax: +385 (51) 686166
}

\author{
InTech China \\ Unit 405, Office Block, Hotel Equatorial Shanghai \\ No.65, Yan An Road (West), Shanghai, 200040, China \\ 中国上海市延安西路65号上海国际贵都大饭店办公楼405单元 \\ Phone: +86-21-62489820 \\ Fax: $+86-21-62489821$
}


www.intechopen.com 
(C) 2008 The Author(s). Licensee IntechOpen. This chapter is distributed under the terms of the Creative Commons Attribution-NonCommercialShareAlike-3.0 License, which permits use, distribution and reproduction for non-commercial purposes, provided the original is properly cited and derivative works building on this content are distributed under the same license. 